\title{
SURFACES WITH PARALLEL MEAN CURVATURE VECTOR
}

\author{
BY BANG-YEN CHEN \\ Communicated by Philip Hartman, March 20, 1972
}

Let $M$ be a surface immersed in a Riemannian manifold $R^{m}$ of dimension $m$. Let $D$ denote the covariant differentiation of $R^{m}$ and $n$ be a normal vector field on $M$. If we denote by $D^{*} n$ the normal component of $D n$, then $D^{*}$ defines a connection in the normal bundle. A normal vector field $n$ is called parallel if $D^{*} n=0$.

Let $\boldsymbol{H}$ and $\boldsymbol{h}$ denote the mean curvature vector and the second fundamental form of $M$ in $E^{m}$. It is easy to see that minimal surfaces of a euclidean $m$-space $E^{m}$ and minimal surfaces of hyperspheres of $E^{m}$ are surfaces of $E^{m}$ with parallel mean curvature vector, i.e. $D^{*} \boldsymbol{H}=0$. On the other hand, for any analytic function $\varphi \neq 0$ of $z=u+i v$, defined in a neighborhood of the origin in the $(u, v)$-plane, and constants $\alpha, \beta$ with $\alpha>0$, Hoffman [3], [4] proved that, up to euclidean motions and isothermal coordinate $E(u, v)$, locally there exists one and only one surface in $E^{4}$, denoted by $M(\varphi, \alpha, \beta)$, with parallel mean curvature vector $\boldsymbol{H}$ such that $\alpha=|H|$, and $\varphi=\varphi_{3}, \beta \varphi=\varphi_{4}$ where $\varphi_{3}$ and $\varphi_{4}$ are given in the Lemma of [3]. These surfaces are easy to check that they are contained in either an affine 3-space or an ordinary 3-sphere of $E^{m}$ and they are neither minimal surfaces in $E^{m}$ nor minimal surfaces of hyperspheres of $E^{m}$. Hence, the following problems seem to be interesting.

Problem I. Let $M$ be a surface immersed in a euclidean $m$-space $E^{m}$ with parallel mean curvature vector. If $M$ is neither a minimal surface of $E^{m}$ nor a minimal surface of a hypersphere of $E^{m}$, is $M$ contained either in an affine 3-space of $E^{m}$ or in an ordinary 3-sphere of $E^{m}$ ?

Problem II. If the answer to Problem $I$ is in the affirmative, is $M$ given locally by one of the surfaces $M(\varphi, \alpha, \beta)$ ?

The main purpose of this paper is to announce the following results. The details will appear elsewhere.

THEOREM I. The answer to Problem I is in the affirmative.

THEOREM II. The answer to Problem II is in the affirmative.

From theorem I we have the following corollaries.

COROLlaRY 1. Let $M$ be a surface immersed in an $m$-sphere $S^{m}$ with

AMS 1970 subject classifications. Primary 53A05, 53A10.

Key words and phrases. Parallel mean curvature vector, minimal surfaces, normal curvature, $M(\varphi, \alpha, \beta)$. 
parallel mean curvature vector. If $M$ is neither a minimal surface of $S^{m}$ nor a minimal surface of a small $(m-1)$-sphere of $S^{m}$, then $M$ must be a surface in a (small or great) 3-sphere of $S^{m}$ with constant mean curvature.

This corollary follows immediately from Theorem I by imbedding $S^{m}$ as a hypersphere of $E^{m+1}$.

COROLlaRY 2. Let $M$ be a compact surface in $E^{m}$ with parallel mean curvature vector and vanishing Gauss curvature. Then $M$ is a product surface of two plane circles.

This corollary follows immediately from Theorem 1 of [2] and a result of Lawson [5].

COROLlary 3. Let $M$ be a complete surface in $E^{m}$ with parallel mean curvature vector. If the Gauss curvature does not change sign, then $M$ is one of the following surfaces:

(i) a minimal surface of $E^{m}$,

(ii) a minimal surface of a hypersphere of $E^{m}$,

(iii) a product surface of two plane circles, or

(iv) a product surface of a straight line and a plane circle.

This corollary follows immediately from Theorem 2 of [3] and Theorem I.

Theorem II follows from Theorem I and the construction of $M(\varphi, \alpha, \beta)$ and Theorem $I$ is based on the following lemmas.

LEMMA 1. Let $M$ be a surface immersed in $E^{m}$ with parallel mean curvature vector and let $\boldsymbol{R}^{N}$ be the curvature tensor of the normal bundle. If $\boldsymbol{H} \neq 0$, then either $M$ is a minimal surface of a hypersphere of $E^{m}$ or $M$ has vanishing normal curvature tensor, i.e. $R^{N}=0$.

LEMMA 2. Let $M$ be a surface in $E^{m}$ with parallel mean curvature vector and vanishing normal curvature tensor. Then $M$ is contained in an affine 4-space of $E^{m}$.

\section{REFERENCES}

1. B.-Y.Chen, On the mean curvature of submanifolds of euclidean space, Bull. Amer. Math. Soc. 77 (1971), 741-743.

2. B.-Y.Chen and G. D. Ludden, Rigidity theorems for surfaces in euclidean space, Bull. Amer. Math. Soc. 78 (1972), 72-73.

3. D. A. Hoffman, Surfaces in constant curvature manifolds with parallel mean curvature vector field, Bull. Amer. Math. Soc. 78 (1972), 247-250.

4. , Surfaces of constant mean curvature in constant curvature manifolds (to appear).

5. H. B. Lawson, Jr., Minimal varieties in constant curvature manifolds, Thesis, Stanford University, Stanford, Calif., 1968.

6. E. A. Ruh, Minimal immersions of 2-spheres in $S^{4}$, Proc. Amer. Math. Soc. 28 (1971), 219-222. MR 42 \# 6761 . 48823

Department of Mathematics, Michigan State University, East Lansing, Michigan 